\title{
Ab Initio Study of Hot Carriers in the First Picosecond after Sunlight Absorption in Silicon
}

\author{
Marco Bernardi, ${ }^{1,2}$ Derek Vigil-Fowler, ${ }^{1,2}$ Johannes Lischner, ${ }^{1,2}$ Jeffrey B. Neaton, ${ }^{1,2,3,4,{ }^{*}}$ and Steven G. Louie ${ }^{1,2, \uparrow}$ \\ ${ }^{1}$ Department of Physics, University of California, Berkeley, California 94720-7300, USA \\ ${ }^{2}$ Materials Science Division, Lawrence Berkeley National Laboratory, Berkeley, California 94720, USA \\ ${ }^{3}$ Molecular Foundry, Lawrence Berkeley National Laboratory, Berkeley, California 94720, USA \\ ${ }^{4}$ Kavli Institute for Energy Nanosciences, Berkeley, California 94720, USA
}

(Received 21 January 2014; published 26 June 2014)

\begin{abstract}
Hot carrier thermalization is a major source of efficiency loss in solar cells. Because of the subpicosecond time scale and complex physics involved, a microscopic characterization of hot carriers is challenging even for the simplest materials. We develop and apply an ab initio approach based on density functional theory and many-body perturbation theory to investigate hot carriers in semiconductors. Our calculations include electron-electron and electron-phonon interactions, and require no experimental input other than the structure of the material. We apply our approach to study the relaxation time and mean free path of hot carriers in $\mathrm{Si}$, and map the band and $\mathbf{k}$ dependence of these quantities. We demonstrate that a hot carrier distribution characteristic of Si under solar illumination thermalizes within $350 \mathrm{fs}$, in excellent agreement with pump-probe experiments. Our work sheds light on the subpicosecond time scale after sunlight absorption in $\mathrm{Si}$, and constitutes a first step towards ab initio quantification of hot carrier dynamics in materials.
\end{abstract}

PACS numbers: 78.56.-a, 71.20.Mq, 78.47.db, 88.40.H-

Single-junction solar cells based on crystalline $\mathrm{Si}$ are rapidly approaching the Shockley-Queisser efficiency limit $[1,2]$. While the Carnot efficiency of $\sim 95 \%$ sets the ultimate limit for solar energy conversion at room temperature, practical efficiency limits in ordinary photovoltaic (PV) solar cells are significantly lower; e.g., the ShockleyQueisser limit for Si is close to $30 \%$ [2]. The main factors limiting efficiency are carrier thermalization and absorption losses [3,4]. For the case of $\mathrm{Si}$ under AM1.5 solar illumination [5], nearly $25 \%$ of incident solar energy is lost to heat as the nonequilibrium ("hot") carriers generated by sunlight absorption thermalize to the edges of the band gap. Not only is hot carrier thermalization the main source of loss in most PV materials, it is also difficult to prevent, control, and understand with microscopic detail due to the subpicosecond time scale typical of hot carrier relaxation [6]. This scenario is common to other technologies employing hot carriers, including electronics, optoelectronics, and renewable energy devices beyond PV [7-11].

The leading mechanisms involved in hot carrier thermalization consist of inelastic electron-phonon (e-ph) and electron-electron $(e-e)$ scattering processes [12]. Relaxation times for $e$-ph and $e$-e interactions in semiconductors have been studied extensively by model Hamiltonians with selected phonon modes, simplified electronic band structures, deformation potentials, and/or empirical pseudopotentials [13-18]. Hot carrier dynamics in semiconductors has been investigated experimentally using pump-probe optical measurements $[19,20]$.

This work has two main goals. First, we present an $a b$ initio approach based on density functional theory
(DFT) and many-body perturbation theory to investigate hot carriers in materials. Second, we apply this framework to study hot carrier thermalization at subpicosecond time scale after sunlight absorption in the simplest and most commonly used material in PV, namely Si. Our approach improves over previous methods employed to study hot carriers [16-18], and is widely applicable. In particular, our formalism allows one to (1) treat all phonon modes on the same footing by using full phonon dispersion curves rather than single phonon modes, (2) resolve hot carrier relaxation times, mean free paths, and dynamics for different bands and $\mathbf{k}$ points in the Brillouin zone (BZ), and (3) compute the $e$-ph coupling matrix elements on fine grids in the $\mathrm{BZ}$ by combining $a b$ initio pseudopotentials and wave functions. For example, within our $a b$ initio framework there is no need to employ deformation potentials to describe $e$-ph scattering rates. This work does not aim to suggest strategies to mitigate thermalization losses in solar cells [21,22], but rather aims to study hot carriers using accurate $a b$ initio calculations.

We compute hot carrier relaxation times and mean free paths in Si, and map these quantities in the BZ. Our calculations indicate that hot carrier thermalization in $\mathrm{Si}$ is dominated by phonon emission processes with a time scale of $\sim 100$ fs for carriers near the band edges, while faster relaxation times of $\sim 10 \mathrm{fs}$ are found at higher energies away from the band edges. We predict that an initial carrier distribution characteristic of Si under AM1.5 solar illumination thermalizes within $350 \mathrm{fs}$, in excellent agreement with pump-probe experiments.

We carry out calculations on a unit cell of $\mathrm{Si}$ with a lattice parameter of $5.4 \AA$. The ground-state electronic 
structure is computed within the local density approximation (LDA) of DFT using the QUANTUM ESPRESSO code $[23,24]$. Norm-conserving pseudopotentials are employed [25], and a kinetic energy cutoff of $45 \mathrm{Ry}$ is used for the plane-wave basis set. Lattice-dynamical properties are computed using density functional perturbation theory [26]. We employ the EPW code [27] to compute the imaginary part of the $e$-ph self-energy $\operatorname{Im}\left(\Sigma_{n, \mathbf{k}}^{e-p h}\right)$ for the Bloch state of energy $\epsilon_{n, \mathbf{k}}$ at band $n$ and $\mathbf{k}$ point in the BZ [28]:

$$
\operatorname{Im}\left(\Sigma_{n \mathbf{k}}^{e-\mathrm{ph}}\right)=\sum_{m, \lambda, \mathbf{q}}\left|g_{n, m, \mathbf{k}}^{\lambda, \mathbf{q}}\right|^{2} \operatorname{Im}\left[\frac{N_{\lambda, \mathbf{q}}+1-f_{m, \mathbf{k}}}{\epsilon_{n, \mathbf{k}}-\epsilon_{m, \mathbf{k}+\mathbf{q}}-\hbar \omega_{\lambda, \mathbf{q}}-i \eta}+\frac{N_{\lambda, \mathbf{q}}-f_{m, \mathbf{k}}}{\epsilon_{n, \mathbf{k}}-\epsilon_{m, \mathbf{k}+\mathbf{q}}+\hbar \omega_{\lambda, \mathbf{q}}-i \eta}\right],
$$

where $\hbar \omega_{\lambda, \mathbf{q}}$ is the energy of a phonon with polarization $\lambda$ and wave vector $\mathbf{q}$ in the $\mathrm{BZ}, f_{m, \mathbf{k}}$ and $N_{\lambda, \mathbf{q}}$ are Fermi and Bose occupation numbers, respectively, evaluated here at $300 \mathrm{~K}$, and $\eta$ is a small Lorentzian broadening. In Eq. (1), the key quantities are the $e$-ph matrix elements, defined as

$$
g_{n, m, \mathbf{k}}^{\lambda, \mathbf{q}}=\left\langle\Psi_{m, \mathbf{k}+\mathbf{q}}\left|\partial_{\lambda, \mathbf{q}} V\right| \Psi_{n, \mathbf{k}}\right\rangle,
$$

where $\Psi_{n, \mathbf{k}}$ is a Kohn-Sham wave function, and $\partial_{\lambda, \mathbf{q}} V$ is the variation of the Kohn-Sham potential for a unit displacement of the nuclei along the phonon mode of polarization $\lambda$ and wave vector $\mathbf{q}$. The $e$-ph relaxation times are computed from the imaginary part of the self-energy as $\tau_{n, \mathbf{k}}^{e-\mathrm{ph}}=\left[\operatorname{Im}\left(\Sigma_{n, \mathbf{k}}^{e-\mathrm{ph}}\right)\right]^{-1} \hbar / 2$. We first compute the electronic and vibrational states and the associated $e$-ph matrix elements on $12 \times 12 \times 12 \mathbf{k}$ and $6 \times 6 \times 6 \mathbf{q}$ point grids using DFT. We then interpolate the quantities needed to evaluate the $e$-ph self-energy on significantly finer grids of $50 \times 50 \times 50 \mathbf{k}$ and $\mathbf{q}$ points, using an interpolation procedure based on Wannier functions [29,30] implemented in the EPW code [27]. Our Wannier functions consist of four $s p^{3}$ orbitals on each $\mathrm{Si}$ atom, leading to the wannierization of 4 valence and 4 conduction bands. We carry out fullfrequency $G W$ calculations [31] using the BERKELEY-GW code [32] to compute the imaginary part of the $e$-e selfenergy, $\operatorname{Im}\left(\Sigma_{n, \mathbf{k}}^{e-e}\right)$, and the associated $e-e$ relaxation time $\tau_{n, \mathbf{k}}^{e-e}=\left[\operatorname{Im}\left(\Sigma_{n, \mathbf{k}}^{e-p h}\right)\right]^{-1} \hbar / 2$. Kinetic energy cutoffs of 5 and $10 \mathrm{Ry}$ are used, respectively, for the screened and bare Coulomb interactions, and 96 empty bands are used to compute the dielectric screening and the Green's function [33]. Finally, we study the time evolution of the electronic occupation number $f_{n, \mathbf{k}}(t)$ for hot carriers. At each time $t$, the occupations are time stepped using the relaxation-time approximation of the Boltzmann equation:

$$
\frac{d f_{n, \mathbf{k}}(t)}{d t}=-\frac{f_{n, \mathbf{k}}(t)-f_{n, \mathbf{k}}\left(t_{\mathrm{th}}\right)}{\tau_{n, \mathbf{k}}},
$$

where $t_{\text {th }}$ is the time after which thermalization is complete, and the relaxation time $\tau_{n, \mathbf{k}}$ is obtained by combining the $e$-ph and $e$-e relaxation times via the Matthiessen rule, $\left(\tau_{n, \mathbf{k}}\right)^{-1}=\left(\tau_{n, \mathbf{k}}^{e-e}\right)^{-1}+\left(\tau_{n, \mathbf{k}}^{e-p h}\right)^{-1}$.
Figure 1(a) shows the imaginary part of the $e$-ph and $e-e$ self-energies in the energy range of relevance for solar excitation [5]. We find that the $e$-ph contribution to the imaginary part of the self-energy is significantly larger than the $e$ - $e$ contribution throughout the energy range of interest. In addition, $\operatorname{Im}\left(\Sigma^{e-p h}\right)$ shows a strong energy dependence with trends similar to the electronic density of states (DOS), consistent with the fact that in this case the DOS regulates the phase space for $e$-ph scattering [14]. The hot electron scattering rates in Fig. 1(a) are in very good agreement with previous calculations from Fischetti et al. using empirical pseudopotentials [16,17]. Our ability to resolve $e$-ph processes on fine grids in the BZ leads to additional details not observed in previous calculations. For example, we observe the presence of a feature with lower $\operatorname{Im}\left(\Sigma^{e-p h}\right)$ values (equivalently, lower scattering rates) due to the $L$ valley at $\sim 1 \mathrm{eV}$ above the conduction band edge, as well as an inherent spread of the scattering rate for a fixed energy due to the $\mathbf{k}$ dependence of the $e$-ph matrix elements. The $e$-e selfenergy is smaller than $10 \mathrm{meV}$ within $3 \mathrm{eV}$ of the midpoint of the band gap, and vanishes for energies between $E_{g}$ below the valence band maximum (VBM) and $E_{g}$ above the conduction band minimum (CBM), where $E_{g}=1.15 \mathrm{eV}$ is the band gap of Si. These trends can be understood from the fact that our $G W$ self-energy encodes information related to the electron-hole processes of a quasiparticle at zero temperature. Hence, the lowest-energy electron-hole
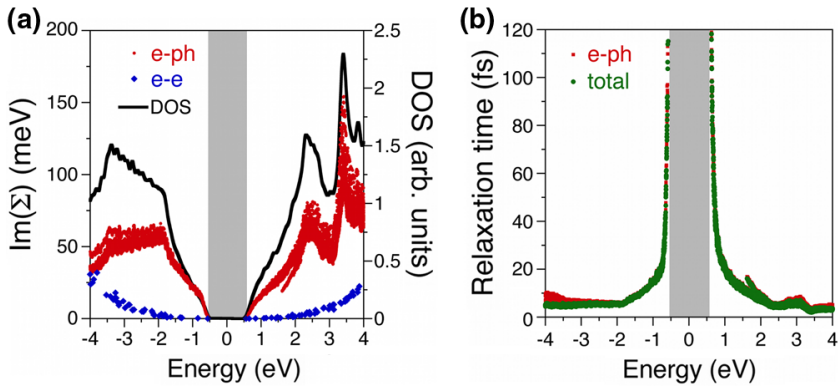

FIG. 1 (color online). (a) Imaginary part of the $e$-ph and $e-e$ self-energies, and the electronic DOS. (b) Relaxation times associated with the $e$-ph self-energy alone and with both the $e$-ph and $e$ - $e$ self-energies (curve labeled as "total"). The zero of the energy axis is placed at the midpoint of the band gap (shown as a shaded area). 
channels captured here consist of interband impact ionization and Auger recombination processes, the onset of which is $\sim E_{g}$ below the VBM and above the CBM, respectively, for hole and electron quasiparticles. Because of the low carrier concentrations excited by solar irradiation-typically less than $10^{17} \mathrm{~cm}^{-3}$ carriers-intraband $e-e$ processes in the hot carrier gas are expected to play a minor role [19]. We conclude that for Si under solar excitation, hot carrier thermalization is dominated by phonon emission processes, while impact ionization processes are less effective due to the $1.15 \mathrm{eV}$ gap of $\mathrm{Si}$ and the low carrier concentration.

Our data in Fig. 1(b) further indicate that hot carrier relaxation near the band edges is characterized by relaxation times of 50-100 fs, while faster relaxation times of 10-20 fs are found at energies more than $300 \mathrm{meV}$ away from the band edges, for both electrons and holes. The inclusion of $e-e$ interactions changes the relaxation times only very slightly [Fig. 1(b)]. Within an optical phonon energy $(\sim 60 \mathrm{meV}$ in $\mathrm{Si}$ ) of the $\mathrm{VBM}$ and $\mathrm{CBM}$, the relaxation times become larger than $100 \mathrm{fs}$ due to the lack of phonon emission processes. In this energy range thermalization is typically fast and mediated by intraband $e$-e processes not included here. The rate of hot carrier thermalization is thus limited by the slowest $e$-ph relaxation times of 50-100 fs at energies of $\sim 200 \mathrm{meV}$ away from the band edges.

The rate of $e$-ph processes responsible for hot carrier thermalization can be mapped in the BZ by analyzing $\operatorname{Im}\left(\Sigma_{n, \mathbf{k}}^{e-p h}\right)$ along high-symmetry directions. Figure 2 shows the electronic band structure of Si combined with information related to $\operatorname{Im}\left(\Sigma_{n, \mathbf{k}}^{e-p h}\right)$ on a fine $\mathbf{k}$ grid. We observe a strong $\mathbf{k}$ and band dependence of $\operatorname{Im}\left(\Sigma_{n, \mathbf{k}}^{e-\mathrm{ph}}\right)$ and the associated $e$-ph relaxation time $\tau_{n, \mathbf{k}}^{e-\mathrm{ph}}$. Energy windows with several bands result in shorter relaxation times due to a larger number of possible transitions accompanying phonon emission from hot carriers. The $\mathbf{k}$ dependence of the relaxation time is further dictated by the $\mathbf{k}$ dependence of the $e$-ph matrix elements.

Maps of $e$-ph scattering rates in the BZ may prove crucial to devise new strategies for hot carrier utilization $[11,21]$.

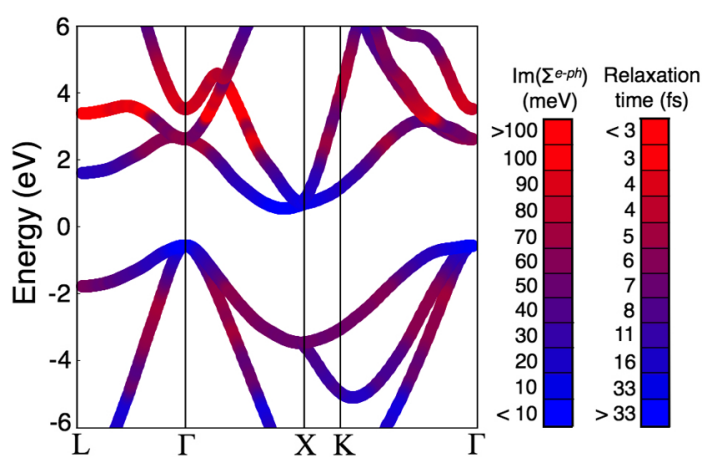

FIG. 2 (color online). The band structure of $\mathrm{Si}$, together with a color map of the $\operatorname{Im}\left(\Sigma^{e-p h}\right)$ and relaxation time.
By relating high-symmetry directions in the $\mathrm{BZ}$ to real space, information regarding hot carrier relaxation along different crystallographic directions can be retrieved, thus assisting the design of experiments aimed at collecting hot carriers. We combine the semiclassical velocities $v_{n, \mathbf{k}}=$ $\hbar^{-1} \partial E_{n, \mathbf{k}} / \partial \mathbf{k}$ with the $e$-ph relaxation times to obtain the $e$-ph mean free paths (MFPs) $L_{n, \mathbf{k}}=v_{n, \mathbf{k}} \cdot \tau_{n, \mathbf{k}}$. The $e$-ph MFPs represent the average distance hot carriers travel before emitting (or absorbing) a phonon. When collected at distances shorter than the MFP, the energy and momentum distributions of hot carriers are preserved, thus enabling extraction before thermalization $[7,11]$.

Figure 3 shows $e$-ph MFPs for hot electrons and holes in Si along the [100], [110], and [111] directions, obtained by computing $v_{n, \mathbf{k}}$ and $\tau_{n, \mathbf{k}}$ on fine grids, respectively, along the $\Gamma X, \Gamma K$, and $\Gamma L$ directions of the BZ. For hot electrons, we observe MFPs of less than $5 \mathrm{~nm}$ at all energies and for all directions, with the exception of the [100] direction at energies near the CBM for which we predict MFPs of up to $15 \mathrm{~nm}$. Such longer MFPs are obtained for energies up to $0.5 \mathrm{eV}$ above the $\mathrm{CBM}$, and stem from velocities near the bottom of the $X$ valley, combined with relatively long (50-100 fs) relaxation times due to small DOS values near the CBM. Hot holes exhibit overall larger MFPs of up to $15 \mathrm{~nm}$ that are evenly distributed among the different directions. Our results indicate that extraction of hot electrons is best achieved in a thin film of $\mathrm{Si}(100)$ with less than $\sim 10 \mathrm{~nm}$ thickness, combined with excitation energies of $\sim 1.5 \mathrm{eV}$. On the other hand, we predict that hot holes may be extracted from thin films of up to $15 \mathrm{~nm}$ thickness with any orientation for excitation energies in the $1.2-3 \mathrm{eV}$ range [34].

We use the data obtained so far to simulate the ultrafast thermalization of $10^{17} \mathrm{~cm}^{-3}$ hot carriers generated by sunlight absorption in $\mathrm{Si}$ [35]. First, the initial hot carrier occupations $f_{n, \mathbf{k}}(t=0)$ imparted by sunlight are estimated by combining the computed density of states $D(E)$ with experimental data for optical absorption in Si [36] and AM1.5 solar radiation [5]:
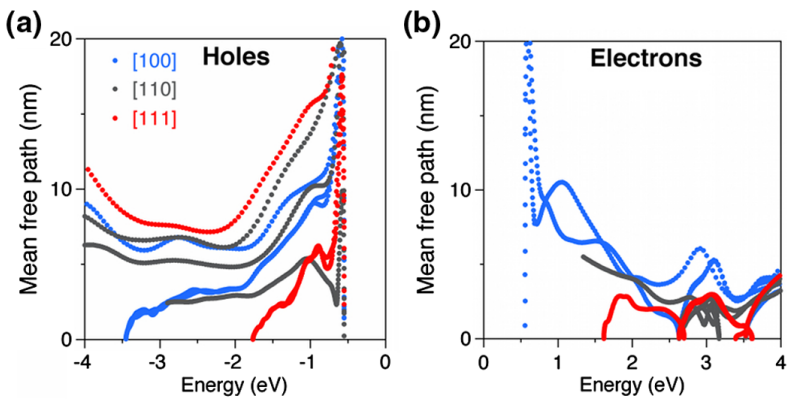

FIG. 3 (color online). Mean free path as a function of energy for hot holes (a) and electrons (b) in $\mathrm{Si}$, shown for the three crystallographic directions [100], [110], and [111]. The curves combine data from multiple bands. The zero of the energy axis is placed at the midpoint of the band gap. 
$f_{n, \mathbf{k}}(t=0) \propto \int_{0}^{4 \mathrm{eV}} d \omega D\left(\epsilon_{n, \mathbf{k}} \pm \omega\right) J_{\mathrm{ph}}(\omega) \alpha(\omega) \theta\left(\omega \pm \epsilon_{n, \mathbf{k}}\right)$,

where $\epsilon_{n, \mathbf{k}}$ is the energy of the hot carrier, $\omega$ is the photon energy, $J_{\mathrm{ph}}$ is the solar irradiance, $\alpha$ is the absorption spectrum of $\mathrm{Si}, \theta$ is the theta step function, and the upper sign refers to holes while the lower sign refers to electrons. In Eq. (4), the band index $n$ runs over the valence and conduction bands for hot holes and electrons, respectively, and the equation estimates the number of interband transitions generating hot carriers. The occupations are then time stepped using Eq. (3), with the occupations $f_{n, \mathbf{k}}\left(t_{\text {th }}\right)$ after thermalization consisting of a Fermi-Dirac distribution centered at an electron quasi-Fermi level such that $10^{17} \mathrm{~cm}^{-3}$ carriers are still present after thermalization. This approach is consistent with the fact that the time scale for thermalization is much shorter than the $\sim 100 \mu \mathrm{s}$ interband recombination time in $\mathrm{Si}$ [37].

Figures 4(a) and 4(b) show the hot carrier population, $P(E)=f(E) D(E)$, for holes and electrons at times $t=0$ and after thermalization. Since the states within $300 \mathrm{meV}$ of the band edges possess relaxation time one order of magnitude longer than states at higher energy within the bands, we expect the thermalization rate to be limited by the slower dynamics of hot carriers near the band edges.
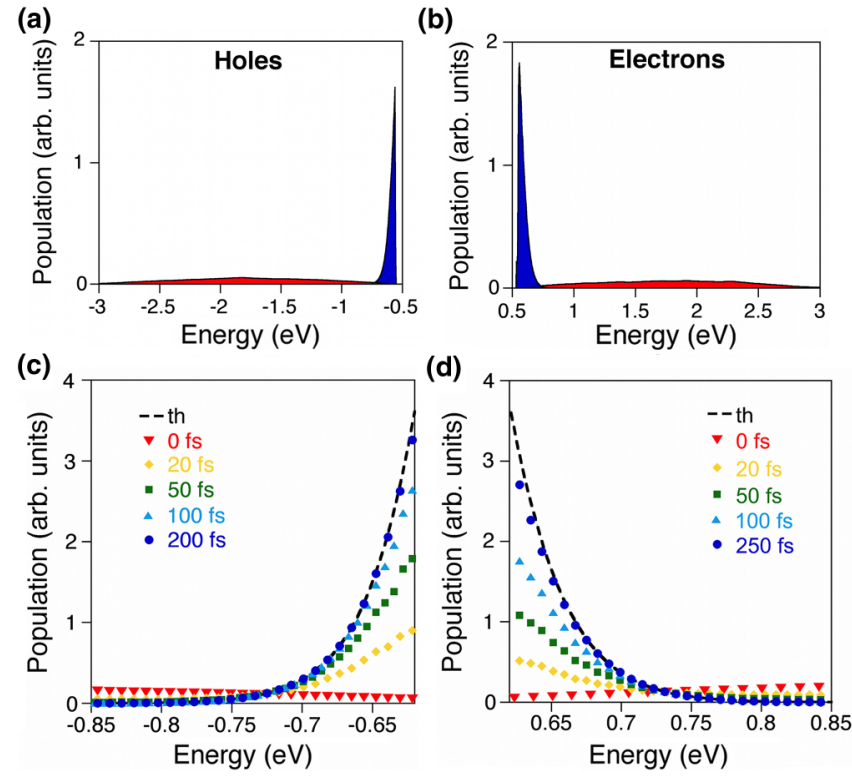

FIG. 4 (color online). Initial hot carrier population (red curve) after photoexcitation with AM1.5 sunlight, and final population after thermalization (blue curve) for $10^{17} \mathrm{~cm}^{-3}$ holes (a) and electrons (b) in Si. The zero of the energy axis is placed at the midpoint of the band gap. The hot hole (c) and electron (d) population dynamics is shown for energies within $300 \mathrm{meV}$ of the VBM and CBM, respectively. Energies within $60 \mathrm{meV}$ of the band edges are not shown. The dashed line labeled as "th" is the occupation after thermalization is complete.
The thermalization of hot carriers in this energy range is detailed in Figs. 4(c) and 4(d), showing the carrier populations as a function of time together with the final Fermi-Dirac distribution for thermalized carriers. The energy range within $\sim 60 \mathrm{meV}$ of the band edges is excluded as thermalization there is not contributed by $e$-ph processes, as noted above. Our results show that thermalization near the band edges completes in $\sim 200$ fs for holes and $\sim 250 \mathrm{fs}$ for electrons. Thermalization completes within $50 \mathrm{fs}$ at higher energies within the bands (not shown), consistent with the faster relaxation times of $\sim 10 \mathrm{fs}$ predicted for energies away from the band edges.

We compare our results with experiments for hot electron thermalization in Si. Doany et al. [20] performed timeresolved reflectivity measurements on $\mathrm{Si}$, and obtained a thermalization time of $350 \mathrm{fs}$ for $10^{17} \mathrm{~cm}^{-3}$ hot electrons excited with $0.8 \mathrm{eV}$ excess energy above the CBM. Our results are in excellent agreement with their measurements. In fact, we predict thermalization times of $250 \mathrm{fs}$ at energies near the conduction band edge, combined with a loss of $\sim 60 \mathrm{meV}$ (the energy of an optical phonon in $\mathrm{Si}$ ) every $\sim 10$ fs at higher energies. For hot carriers with an energy of $0.8 \mathrm{eV}$ above the CBM as in Ref. [20], we thus predict a loss of $600 \mathrm{meV}$ in $100 \mathrm{fs}$ to relax the hot carriers to $200 \mathrm{meV}$ above the CBM, followed by thermalization in $250 \mathrm{fs}$. This yields a total thermalization time of approximately $350 \mathrm{fs}$, as in the experiments in Ref. [20]. Our results are also consistent with thermalization times extracted from transport measurements in combination with Monte Carlo calculations $[18,20,38]$, predicting thermalization within $250 \mathrm{fs}$ near the CBM. Since the vast majority of hot electrons generated by sunlight in $\mathrm{Si}$ are found within $1-2 \mathrm{eV}$ above the CBM, we conclude that hot electron thermalization under sunlight illumination in Si occurs over $\sim 350$ fs. Our calculations indicate a similar time scale for hot hole thermalization.

In summary, our work demonstrates that hot carrier thermalization losses in $\mathrm{Si}$ under sunlight illumination occur at ultrafast ( $\sim 350 \mathrm{fs})$ time scale and are dominated by phonon emission from hot carriers with energies near the band edges. Our $a b$ initio approach can provide microscopic information regarding hot carriers that would be difficult to extract from experiment, such as the energy and momentum dependence of hot carrier relaxation times and MFPs. Our work sheds light on the microscopic origin of the main efficiency loss in Si solar cells, and paves the way to $a b$ initio studies of hot carriers in materials for renewable energy.

M. B. thanks Sinisa Coh for discussion. This research was supported by the SciDAC Program on Excited State Phenomena in Energy Materials funded by the U.S. Department of Energy, Office of Basic Energy Sciences and of Advanced Scientific Computing Research, under Contract No. DE-AC02-05CH11231 at Lawrence Berkeley National Laboratory, which provided for algorithm and 
code developments and simulations; and by the National Science Foundation under Grant No. DMR 10-1006184 which provided for basic theory and formalism. Work at the Molecular Foundry was supported by the Office of Science, Office of Basic Energy Sciences, of the U.S. Department of Energy under Contract No. DE-AC02-05CH11231. S. G. L. acknowledges support of a Simons Foundation Fellowship in Theoretical Physics. This research used resources of the National Energy Research Scientific Computing Center, which is supported by the Office of Science of the U.S. Department of Energy.

*Corresponding author.

jbneaton@lbl.gov

Corresponding author. sglouie@berkeley.edu

[1] D. Ginley, M. A. Green, and R. Collins, MRS Bull. 33, 355 (2008).

[2] W. Shockley and H. J. Queisser, J. Appl. Phys. 32, 510 (1961).

[3] A. Polman and H. A. Atwater, Nat. Mater. 11, 174 (2012).

[4] P. Wurfel, Physica (Amsterdam) 14E, 18 (2002).

[5] The AM1.5G spectrum was taken from the NREL website: http://rredc.nrel.gov/solar/spectra/am1.5 and integrated with the trapezoid rule. The website was accessed in October 2013.

[6] C. V. Shank and B.P. Zakharchenya, Spectroscopy of Nonequilibrium Electrons and Phonons (Elsevier, Amsterdam, 1992).

[7] W. A. Tisdale, K. J. Williams, B. A. Timp, D. J. Norris, E. S. Aydil, and X.-Y. Zhu, Science 328, 1543 (2010).

[8] N. M. Gabor, J.C. Song, Q. Ma, N. L. Nair, T. Taychatanapat, K. Watanabe, T. Taniguchi, L. S. Levitov, and P. Jarillo-Herrero, Science 334, 648 (2011).

[9] J. Iveland, L. Martinelli, J. Peretti, J.S. Speck, and C. Weisbuch, Phys. Rev. Lett. 110, 177406 (2013).

[10] H. Chalabi and M. L. Brongersma, Nat. Nanotechnol. 8, 229 (2013).

[11] S. Mukherjee, F. Libisch, N. Large, O. Neumann, L. V. Brown, J. Cheng, J. B. Lassiter, E. A. Carter, P. Nordlander, and N. J. Halas, Nano Lett. 13, 240 (2013).

[12] H. Haug and A.-P. Jauho, Quantum Kinetics in Transport and Optics of Semiconductors (Springer-Verlag, Berlin, Heidelberg, 2008).

[13] M. Cardona, Solid State Commun. 133, 3 (2005).

[14] G. D. Mahan, Condensed Matter in a Nutshell (Princeton University Press, Princeton, 2010).

[15] R. Jalabert and S. Das Sarma, Phys. Rev. B 41, 3651 (1990).

[16] M. Fischetti and S. Laux, Monte Carlo Simulation of Electron Transport in Si: The First 20 Years, in Proceedings of the 26th European Solid State Device Research Conference, 1996 (ESSDERC'96), edited by G. Baccarani and M. Rudan (IEEE, Bologna, 1996), pp. 813-820.

[17] M. V. Fischetti and S. E. Laux, Phys. Rev. B 38, 9721 (1988).

[18] C. Jacoboni and L. Reggiani, Rev. Mod. Phys. 55, 645 (1983).
[19] J. Shah, Ultrafast Spectroscopy of Semiconductors and Semiconductor Nanostructures (Springer, Berlin, 1999), Vol. 115

[20] F. Doany and D. Grischkowsky, Appl. Phys. Lett. 52, 36 (1988).

[21] G. Conibeer, N. Ekins-Daukes, J.-F. Guillemoles, D. Kőnig, E.-C. Cho, C.-W. Jiang, S. Shrestha, and M. Green, Sol. Energy Mater. Sol. Cells 93, 713 (2009).

[22] G. Conibeer, D. König, M. Green, and J. Guillemoles, Thin Solid Films 516, 6948 (2008).

[23] D. M. Ceperley and B. J. Alder, Phys. Rev. Lett. 45, 566 (1980): J. P. Perdew and A. Zunger, Phys. Rev. B 23, 5048 (1981).

[24] P. Giannozzi, S. Baroni, N. Bonini, M. Calandra, R. Car, C. Cavazzoni, D. Ceresoli, G. L. Chiarotti, M. Cococcioni, I. Dabo et al., J. Phys. Condens. Matter 21, 395502 (2009).

[25] N. Troullier and J. L. Martins, Phys. Rev. B 43, 1993 (1991).

[26] S. Baroni, S. de Gironcoli, A. Dal Corso, and P. Giannozzi, Rev. Mod. Phys. 73, 515 (2001).

[27] J. Noffsinger, F. Giustino, B. D. Malone, C.-H. Park, S. G. Louie, and M. L. Cohen, Comput. Phys. Commun. 181, 2140 (2010).

[28] F. Giustino, M. L. Cohen, and S. G. Louie, Phys. Rev. B 76, 165108 (2007).

[29] N. Marzari, A. A. Mostofi, J. R. Yates, I. Souza, and D. Vanderbilt, Rev. Mod. Phys. 84, 1419 (2012).

[30] A. A. Mostofi, J. R. Yates, Y.-S. Lee, I. Souza, D. Vanderbilt, and N. Marzari, Comput. Phys. Commun. 178, 685 (2008).

[31] M. S. Hybertsen and S. G. Louie, Phys. Rev. B 34, 5390 (1986)L. Hedin, Phys. Rev. 139, A796 (1965).

[32] J. Deslippe, G. Samsonidze, D. A. Strubbe, M. Jain, M. L. Cohen, and S. G. Louie, Comput. Phys. Commun. 183, 1269 (2012).

[33] For the $G W$ calculations, the PARATEC code was employed for the ground-state DFT-LDA calculation, and we used the "one shot" $G_{0} W_{0}$ approximation. While a fine sampling of the $\mathrm{BZ}$ was found to be essential to convergence the $e$-ph self-energy, an $8 \times 8 \times 8 \mathbf{k}$ grid was sufficient to converge the $G W e$-e self-energy.

[34] We remark that the large MFPs of $20 \mathrm{~nm}$ found at energy within $60 \mathrm{meV}$ of the VBM and CBM should be disregarded, as they are a consequence of our approximations, as discussed above.

[35] Our choice for the hot carrier density constitutes an upper limit under AM 1.5 illumination, as obtained by combining a carrier optical generation rate $G \approx 10^{21} \mathrm{~cm}^{-3} \mathrm{~s}^{-1}$ and an assumed lifetime for interband recombination (see Ref. [37]) of $\tau_{r} \approx 100 \mu \mathrm{s}$, leading to a steady-state carrier density $G \cdot \tau_{r}=10^{17} \mathrm{~cm}^{-3}$.

[36] M. A. Green, Sol. Energy Mater. Sol. Cells 92, 1305 (2008).

[37] R. Jayakrishnan, S. Gandhi, and P. Suratkar, Mater. Sci. Semicond. Process. 14, 223 (2011).

[38] C. Canali, C. Jacoboni, F. Nava, G. Ottaviani, and A. Alberigi-Quaranta, Phys. Rev. B 12, 2265 (1975). 